\title{
Halley's comet is quite young
}

Three years after the most recent apparition of Halley's Comet, estimates of the time for which the comet has been circulting in something like its present orbit seem to be converging on a few tens of thousands of years.

Those who prefer their astronomy to be spiced with poetic imagery are naturally, and perhaps rightly, inclined to regard comets as wanderers in the Solar System. The justification is the now-conventional view of where comets come from: out there, beyond Pluto, is a collection of objects consisting of interstellar dust cemented together with water-ice and other kinds of ice. Gravitational perturbations of this Oort cloud, perhaps by passing stars, then deflect some members of the cloud downwards to the region of Neptune and Uranus whence, perhaps by the influence of Saturn and Jupiter, some of them may be deflected into the inner Solar System, to appear occasionally, but regularly, in the visible night sky.

The poetic image is apt. The chance that a particular member of the Oort cloud will be captured in this way is only small, while an object captured into the outer Solar System is more likely to be expelled from the Solar System altogether than to finish up in an orbit that carries it near enough to the Sun to be a spectacular apparition every now and again. In this accepted view of the appearance of comets, the existence of the Oort cloud itself is not a firm reality, but is inferred, essentially from calculations of the chance that such an object will be captured into the inner Solar System and from the number of known comets. In the circumstances, many people would be happier if there were more objective evidence for the reality of the Oort cloud.

None of that implies that inner Solar System comets are other than transient. By definition, objects such as these which are noticeable in the sky make spectacular sights because of the material they shed during their relatively close passage of the Sun, which implies that they cannot last forever. Indeed, the rate at which comets such as Halley lose material near perihelion is so great that they cannot have been in their present orbits for very long, either.

So it is a reasonable question to ask how long Halley's comet has been in something like its present orbit. It is not, of course, a new question, but now a group of three Canadian astronomers have produced what may be a more reliable estimate of the age so far of Comet Halley (Jones, J., McIntosh, B.A. \& Hawkes, R.L. Mon. Not. R. astr. Soc. 238, 179; 1989).

Jones and McIntosh, from the University of Western Ontario and the National
Research Council of Canada at Ottawa respectively, have a long-standing interest in the properties of the stream of dust ejected from Halley's comet which are recognizable from the surface of the Earth as the Orionid and $\eta$-Aquarid meteor streams (visible in October and the first week of May respectively).

The two meteor showers consist literally of material lost from Halley's comet which is now spread out in a region ranged about the comet's present orbit, and which the Earth intercepts regularly twice a year. If only it were possible accurately to estimate the total mass of the meteor shower, it would be possible to estimate the amount of mass the comet loses at each apparition much more accurately than can be told even from the accurate direct measurements made in 1986.

This is what Jones, McIntosh and Hawkes have been able to do on the basis of an earlier intricate calculation of the likely form of the meteor stream from Halley (Mon. Not. R. astr. Soc. 235, 673; 1988). Against naive expectation, the dust particles ejected from the comet are not arranged in some kind of cylinder running the length of the comet's orbit, but are in the form of a thick ribbon-like structure whose position is determined not merely by all past positions of the orbit of Halley's comet, but also the influence of radiation pressure on the dust the comet has released in clumps during its infrequent perihelion passages.

This calculation confirmed previous guesses by the tedious if effective way of simulating the fate of particles of different sizes by the effects of gravitation and radiation on 500 test particles in each computer run. The upshot of the modelling is that the ejected particles occupy a ribbon-like region of space which is 4 per cent of an astronomical unit thick and ten times as wide. (An astronomical unit is the diameter of the Earth's orbit, or roughly 300 million $\mathrm{km}$.)

That the seasons for the Orionid and $\eta$ Aquarid meteor showers bear little relation to the times of the year at which the orbit of Halley's comet now crosses the Ecliptic is simply a measure of the relatively high rate at which Halley's orbit is changing under the influence of Jupiter on the comet at aphelion - the most pronounced change of the orbit is a steady rotation about the major axis in a clockwise direction seen from perihelion.

How much meteor mass is locked up in this ribbon? The only objective answer to this question must be the use of radar observations to estimate the amount of ionization left in the atmosphere by meteors in the shower. Among other things, information of that kind makes it possible to calculate the size-distribution of the dust particles in the meteor streams. The upshot of the calculation, in which the greatest uncertainties must stem from the numerical calculations of the dimensions of the ribbon, amounts to $1.3 \times 10^{13} \mathrm{~kg}$.

That is an arresting number in itself, for this amount of dust is a substantial fraction of the likely mass of Halley's comet proper. Although the mass was not accurately determined at the 1986 apparition, Jones et al. estimate from the accurately measured volume of the nucleus of the comet, and from its probable density, that the mass left intact must be only one order of magnitude greater than the dust scattered into the ribbon-shaped alley in which it now travels since Halley's comet was first captured into the inner Solar System. But because most of the comet's mass must consist of ice, not dust, there can hardly be more than two or three times as much dust to come as the quantity already released. Whatever the future for Halley's comet, it cannot be much longer than the time already spent within the orbit of Jupiter.

And how long is that? Jones et al. must necessarily start with some assumptions about the way in which material is lost from the nucleus of the comet at each perihelion passage. They take it that the amount of solar energy absorbed is proportional to the declining surface area of the nucleus and make assumptions about the proportion of this energy used to sublimate cementing ice (which may be expected to diminish with each succeeding passage). Surprisingly, they find that their estimate of the lifetime (so far) of Halley's comet is relatively insensitive to assumptions about the proportion of the comet that consists of ice.

Their conclusion is that the time Halley's comet has spent in the Inner Solar System is a mere 23,000 years, perhaps enough for fewer than 300 revolutions of the orbit. This result agrees well with another calculation that there must have been a close encounter between the comet and Jupiter something like 20,000 years ago. Not often do such long chains of inference come out so well.

John Maddox 